\title{
Growing wetting films: An x-ray study
}

\author{
M. Strzelczyk, P. Müller-Buschbaum, M. Tolan, and W. Press \\ Institut für Experimentalphysik, Christian-Albrechts-Universität zu Kiel, Leibnizstrasse 19, 24118 Kiel, Germany
}

(Received 26 June 1995; revised manuscript received 10 August 1995)

\begin{abstract}
The growth kinetics of thin liquid films, completely wetting a solid substrate, are studied. The film thickness is perturbed and reduced by a short thermal pulse. Subsequently the time dependence of the growth process was monitored by $\mathrm{x}$-ray reflectivity measurements in the region of total external reflection. The examined sample systems are $\mathrm{CBrCl}_{3}$ films on top of silicon, germanium, and glass wafers and $\mathrm{CBrCl}_{3}$ on glass/gold and glass/silver substrates. The observed growth kinetics of the wetting layer are discussed in the framework of two models that were adapted to the experimental conditions, particularly the finite temperature stability of the experimental setup. From the growth law $l(t) \propto 1-\exp (-t / \tau)^{n}$ fitted to the data, time constants $\tau$ and dynamic exponents $n$ are determined. $n$ depends on the dimension of the growth mechanism. Various systems with quite different substrateadsorbate interactions can be explained quantitatively within this model.
\end{abstract}

\section{INTRODUCTION}

In recent years a large number of studies have focused on wetting phenomena (for a review see de Gennes ${ }^{1}$ or Dietrich $^{2}$ ). Most of these papers deal with the properties of wetting layers which are in thermodynamic equilibrium with their surroundings. The growth of liquid films is a typical case where a film is far away from equilibrium. In the past the case of incomplete wetting and the spreading of droplets on solid surfaces were examined by several authors. $^{3-8}$ In these systems the evolution of the liquid phase takes place in several directions which have different growth characteristics. In the case of complete wetting the film grows only in one direction, which means that the thickness of the liquid layer monotonously increases with time. The theory developed by Lipowsky $^{9}$ yields an expression which explains this behavior quantitatively. Although computer simulations ${ }^{10}$ seem to support this model, it can only explain the growth of a wetting film in an ideal system. For a description of the film evolution under real experimental conditions the Lipowsky model has to be extended slightly. Particularly the finite temperature stability of the setup has to be included.

In this paper the growth of liquid bromotrichloromethane $\left(\mathrm{CBrCl}_{3}\right)$ films on $\mathrm{Si}$ and $\mathrm{Ge}$ wafers covered with their native oxide layers and on top of glass, glass/gold, and glass/silver substrates is investigated. All these substrates provide the case of complete wetting and a stable $\mathrm{CBrCl}_{3}$ film with a thickness in the range of 150-300 A builds up. The thickness was first reduced by a short pulse disturbance caused by a temperature difference between the substrate and the vapor in the sample cell. Subsequently the time dependence of the film formation is monitored by $\mathrm{X}$-ray reflectivity measurements in the region of total external reflection. These measurements are well suited to yield the thickness and the electron-density profile perpendicular to the surface of liquid layer systems. ${ }^{11,12}$

The paper is structured as follows: After the introduction a brief description of the theory of specular x-ray reflectivity is given. Then a discussion of the Lipowsky model and the semiquantitative Kolmogorov approach of an arbitrary growing process follows. Next the experimental setup and the sample system are described. Then measurements with dry substrates and the timedependent measurements are reported. The presentation of the results and a discussion followed by a summary and an outlook finish this paper.

\section{THEORY}

\section{A. X-ray reflectivity}

With the approach of Parrat, ${ }^{13}$ the reflectivity of a layer system can be expressed in terms of a recursive formu$1 a^{14}$

$$
R_{j-1}\left(l_{j-1}\right)=\frac{r_{j-1, j}+R_{j}\left(l_{j}\right) \exp \left(2 i k_{z, j} l_{j}\right)}{1+r_{j-1, j} R_{j}\left(l_{j}\right) \exp \left(2 i k_{z, j} l_{j}\right)},
$$

with the Fresnel reflectivity of one smooth interface

$$
r_{j-1, j}=\frac{k_{z, j-1}-k_{z, j}}{k_{z, j-1}+k_{z, j}} .
$$

Here $R_{j}=E_{j}^{R} / E_{j}^{T}$ is the ratio of the amplitudes of the outgoing and incoming $x$-ray waves. The starting point of the recursion is the substrate which is assumed to be semi-infinite, and therefore $R_{\text {sub }}=0$. In each layer of thickness $l_{j}$ the component of the wave vector normal to the surface $k_{z, j}$ is given by the law of refraction

$$
k_{z, j}=k \sin \Theta_{j} \cong \frac{2 \pi}{\lambda} \sqrt{\Theta^{2}-2\left(\delta_{j}+i \beta_{j}\right)} .
$$

$\lambda$ denotes the wavelength of the $x$-ray radiation. The expression is valid for small angles of incident $\theta$. The optical constants $\delta_{j}$ and $\beta_{j}$ of each layer $j$ (which are proportional to the electron density $\rho_{j}$ ) are defined via the (complex) index of refraction ${ }^{15}$ 


$$
n_{j}=1-\delta_{j}-i \beta_{j} .
$$

The critical angle $\boldsymbol{\Theta}_{c}$ for total external reflection can easily be obtained by the expression $\left(\beta_{j}=0\right)$

$$
\Theta_{c, j}=\sqrt{2 \delta_{j}} \text {. }
$$

In the case of rough surfaces, the Fresnel coefficients in formula (1) have to be replaced by those of rough interfaces $^{14}$

$$
r_{j-1, j}=\frac{\sinh \left[\sigma_{j}(\pi / 2)^{3 / 2}\left(k_{z, j-1}-k_{z, j}\right)\right]}{\sinh \left[\sigma_{j}(\pi / 2)^{3 / 2}\left(k_{z, j-1}+k_{z, j}\right)\right]} G .
$$

From numerical tests it follows that the factor $G$ can be set to 1 for roughnesses $\sigma_{j}$ smaller than $100 \AA .{ }^{16}$

Because of the large density contrast between some of the substrates ( $\mathrm{Au}, \mathrm{Ag}$ ) on one hand and the adsorbate $\left(\mathrm{CBrCl}_{3}\right)$ on the other hand, only slight differences between the reflectivity of the bare and wetted substrates are visible (see Fig. 1). For incident angles $\Theta_{\text {dip }}$ which fulfill the condition $\Theta_{c \text {, adsorbate }} \leq \Theta_{\text {dip }} \leq \Theta_{c \text {, substrate, a dip in }}$ the reflectivity of the wetted substrate appears. ${ }^{17,18}$ In the case of thin liquid layers $(l<500 \AA)$ the intensity reduction in the dip is proportional to the layer thickness of the wetting film. This can be derived for a simple system consisting of a smooth semi-infinite substrate and an adlayer with thickness $l$. Then Eq. (1) yields

$$
R=\left|\frac{r_{0}+r_{1} \exp \left(2 i k_{0} \Theta_{1} l\right)}{1+r_{0} r_{1} \exp \left(2 i k_{0} \Theta_{1} l\right)}\right|^{2} .
$$

The Fresnel reflectivities of the air-layer and layersubstrate interfaces are denoted by $r_{0}$ and $r_{1}$, respectively. Each of them can be decomposed into a modulus and a phase: $r_{j}=\left|r_{j}\right| \exp \left(i \Phi_{j}\right)$, with $j=0$ and 1. Furthermore, $\Theta_{1}=a+i b$ is the (complex) refraction angle inside the layer. $a$ and $b$ are given by

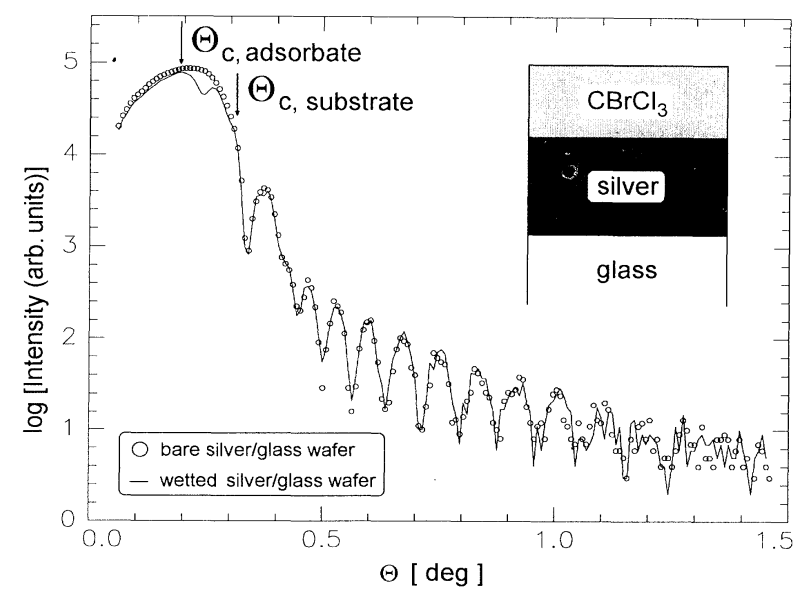

FIG. 1. Measured specular reflectivities of a bare (open circles) and a wetted (solid line) silver/glass wafer. For incident angles $\Theta_{\text {dip }}$, which fulfill the condition $\Theta_{c \text {, adsorbate }} \leq \Theta_{\text {dip }} \leq \Theta_{c \text {, substrate }}$, a dip in the reflectivity of the wetted silver/glass wafer appears. The thickness of the $\mathrm{CBrCl}_{3}$ film is about $280 \AA$. The inset schematically shows the layer system.

$$
\begin{aligned}
& a=\frac{1}{\sqrt{2}}\left[\sqrt{\left(\Theta_{\mathrm{dip}}^{2}-2 \delta\right)^{2}+4 \beta}+\left(\Theta_{\mathrm{dip}}^{2}-2 \delta\right)\right]^{1 / 2}, \\
& b=\frac{1}{\sqrt{2}}\left[\sqrt{\left(\Theta_{\mathrm{dip}}^{2}-2 \delta\right)^{2}+4 \beta}-\left(\Theta_{\mathrm{dip}}^{2}-2 \delta\right)\right]^{1 / 2} .
\end{aligned}
$$

Equation (7) can now be transformed into a relation between the reflectivity $R$ and the film thickness $l$ of the wetting layer:

$$
l=C \frac{R\left(\Theta_{\mathrm{dip}}\right)-1}{R\left(\Theta_{\mathrm{dip}}\right)+1} .
$$

Here $R$ is the reflectivity at the angular position $\Theta_{\text {dip }}$ of the intensity dip, and $C$ a constant which is given by

$$
C=\frac{\lambda}{8 \pi} \frac{1+\left|r_{0}\right|^{2}+2\left|r_{0}\right| \cos \left(\Phi_{0}-\Phi_{1}\right)}{\left|r_{0}\right|\left[a \sin \left(\Phi_{0}-\Phi_{1}\right)+b \sin \left(\Phi_{0}-\Phi_{1}\right)\right]} \text {. }
$$

Consequently, for most of the systems (e.g., $\mathrm{CBrCl}_{3}$ on top of $\mathrm{Au} /$ glass or $\mathrm{Ag} /$ glass) the thickness of the growing wetting film was determined using Eq. (10). This formula only yields the correct thickness if the absolute value of the reflectivity is inserted. However, due to the experimental conditions, only relative $R$ values were measured. Afterwards these relative thicknesses were scaled by fitting the whole reflectivity curve of the stable wetting film to obtain the definite values. Therefore it is possible to determine the actual thicknesses of all investigated systems.

\section{B. Lipowsky model}

The properties of a wetting layer which is in thermal equilibrium at a temperature $T$ are obtained from the free-energy functional ${ }^{19}$

$$
F\{l\}=\int d^{d-1} x\left[1 / 2 \gamma(\nabla l)^{2}+V(l)\right] /\left(k_{B} T\right) .
$$

$\gamma$ is the interfacial tension, $l=l\left(x_{1}, \ldots, x_{d-1}\right)$ is the distance between the $(d-1)$-dimensional interfaces which bounds the wetting film, and $V(l)$ is the free energy per unit area for a layer of constant thickness. In the case of complete wetting $V(l)$ is given by

$$
V(l)=\frac{A}{12 \pi l^{p}}+\delta \mu l .
$$

The first term with the Hamaker constant $A$ favors a larger value of $l$, and the second term with the chemical potential difference $\delta \mu$ between the substrate and the gas phase encourages a smaller value of $l$. As $\delta \mu \rightarrow 0$, the mean thickness of the layer diverges. The exponent $p$ depends on the nature of the van der Waals interaction: $p=\kappa-d-1$, with $\kappa=6$ and 7 for nonretarded and retarded van der Waals forces, respectively.

The dynamic behavior of a wetting film is defined by the Langevin equation

$$
\frac{\partial l(\mathbf{x}, t)}{\partial t}=-\Lambda \frac{\delta F\{l\}}{\delta l}+\zeta .
$$

$\Lambda$ is an Onsager coefficient, and $\xi$ is a Gaussian random force with

$\langle\zeta\rangle=0,\left\langle\xi(\mathbf{x}, t) \xi\left(\mathbf{x}^{\prime}, t^{\prime}\right)\right\rangle=2 \Lambda \delta\left(\mathbf{x}-\mathbf{x}^{\prime}\right) \delta\left(t-t^{\prime}\right)$. 
In the mean-field approximation which is valid for $d>(2+3 p) /(2+p),{ }^{9}$ Eq. (14) can be transformed to

$$
\frac{\partial \bar{l}(t)}{\partial t}=-\left.\frac{\Lambda}{k_{B} T} \frac{\partial V(l)}{\partial l}\right|_{\bar{l}} .
$$

A solution of this differential equation with the potential given by Eq. (13) and $\delta \mu=0$ is

$$
\bar{l}(t)=\left[\frac{p \Lambda A}{12 \pi \psi k_{B} T} t\right]^{\psi},
$$

with $\psi=1 /(2+p)$. This yields $\bar{l}(t) \propto t^{1 / 4}$ for systems with two-dimensional interfaces and nonretarded van der Waals forces.

Although Monte Carlo simulations confirm this growth behavior, ${ }^{10} \mathrm{Eq}$. (17) is not able to describe the time dependence of the wetting layer thickness in reality. In an experiment, $\delta \mu$ is never exactly zero. This means that the thickness of the wetting layer stays finite for $t \rightarrow \infty$.

As a consequence the chemical potential difference $\delta \mu$ in Eq. (13) cannot be set to zero. Instead, the Langevin equation in the mean-field approximation and for constant $\delta \mu \neq 0$ must be written

$$
\frac{d \bar{l}(t)}{d t}=\frac{p \Lambda A}{12 \pi k_{B} T} \bar{l}^{-(l+p)}-\frac{\Lambda \delta \mu}{k_{B} T} .
$$

In the case of two-dimensional interfaces the solution for the dynamic behavior of the film thickness is given by an implicit equation for the time $t(l)$ :

$$
\begin{aligned}
t(l)= & -\frac{k_{B} T}{\Lambda \delta \mu} \bar{l}-\frac{k_{B} T}{6 \Lambda \delta \mu} l_{\infty} \ln \left[\frac{\left(l_{\infty}-\bar{l}\right)^{2}}{l_{\infty}^{2}+l_{\infty} \bar{l}+\bar{l}^{2}}\right] \\
& +\frac{k_{B} T}{\sqrt{3} \Lambda \delta \mu} l_{\infty} \arctan \left[\frac{2 \bar{l}+l_{\infty}}{\sqrt{3} l_{\infty}}\right],
\end{aligned}
$$

with $l_{\infty}=\sqrt[3]{A / 6 \pi \delta \mu}$ the finite value of the wetting layer thickness for $t \rightarrow \infty$. Some further approximations (the third term becomes constant, $l_{\infty} \approx \bar{l}$ in the logarithmic term) which are only valid for $t \gg 0$ lead to an exponential growth law:

$$
\bar{l}(t) \propto l_{\infty}\left\{1-\exp \left(-C\left(l_{\infty}\right) t\right)\right\} .
$$

An expansion of Eq. (19) for short times yields that Eq. (20) is also a good description for the beginning of the film growth. Additionally the evolution of the wetting film might be influenced by the temperature development in the sample cell after the beginning of the thermal disturbance. Of course in an experiment one cannot achieve an instantaneous thermal pulse $\Delta T$ at $t=0$ which vanishes for $t>0$. In order to avoid the solution of a heat transfer equation, the temperature behavior for small $t$ is approximated by the Newton law of cooling, ${ }^{20}$ which means that $\Delta T$ decreases exponentially to a final value $\Delta T_{\infty}$. Accordingly the film thickness is limited by the temperature difference between the substrate and the vapor. Fortunately it turns out that the evolution of the wetting film is independent of the temperature relaxation, since this process is much faster than the growth of the liquid layer.

\section{Kolmogorov model}

The Kolmogorov model ${ }^{21}$ (also often called the Johnson-Mehl-Avrami model ${ }^{22-25}$ ) was developed to describe a first-order phase transformation from a metastable phase $A$ into a stable phase $B$. In this stochastic model, several assumptions are made: (a) the system is infinite, (b) grains of zero size nucleate with a constant rate $\Gamma$, and (c) grains grow isotropically with a constant velocity $v$ until they are impeded by impinging on neighboring domains. Both parameters $\Gamma$ and $v$ are assumed to be time independent. Because the model is completely characterized by these two parameters, the required scaling follows from a dimensional analysis, and it is possible to define a characteristic time scale $\tau$ and a length scale $\xi$ given by

$$
\begin{aligned}
\tau & =\left[C_{d} \Gamma v^{d} /(d+1)\right]^{-1 /(d+1)}, \\
\xi & =(\Gamma / v)^{-1 /(d+1)} .
\end{aligned}
$$

$C_{d}$ is a constant, which depends on the dimension $d$ of the growing mechanism ${ }^{26}$ with $C_{d}=2, \pi$, and $4 \pi / 3$ for $d=1,2$, and 3 . Then, with the approach of Kolmogorov, an explicit expression for the volume fraction $X(t)$ of the transformed material ( $A \rightarrow B$ ) can be given by

$$
X(t)=1-\exp \left[-(t / \tau)^{n}\right],
$$

with the dynamic exponent $n=d+1$.

Equation (22) leads to an exponential law for the growing process of the film: ${ }^{27}$

$$
\bar{l}(t) \propto l_{\infty}\left\{1-\exp \left[-(t / \tau)^{n}\right]\right\} .
$$

In the case of complete wetting the film grows only in the direction perpendicular to the surface, which leads to a dynamic exponent $d=2$.

\section{EXPERIMENTAL SETUP AND SAMPLE SYSTEMS}

The x-ray measurements were performed using a highresolution three-crystal diffractometer (TCD; see Fig. 2). For a detailed discussion of the general setup and the

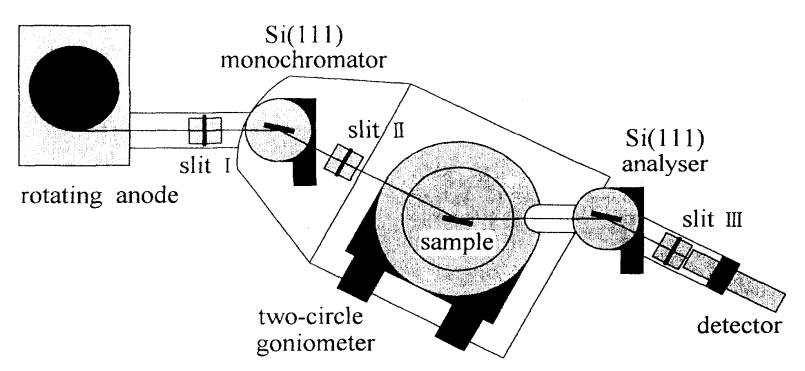

FIG. 2. Setup of a high-resolution three-crystal diffractometer (TCD) with a silicon analyzer and monochromator crystal. 
resolution of the TCD, see Ref. 28. A $12-\mathrm{kW}$ rotatinganode X-ray generator (Rigaku Ru 200) with a copper target was used as source, a $\mathrm{Si}(111)$ monochromator picks out the $\mathrm{Cu} K \alpha$, lines, and a slit selects the $\mathrm{Cu} K \alpha_{1}$ line $(\lambda=1.54056 \AA)$ which impinges onto the sample. The detector unit contains a $\mathrm{Si}(111)$ analyzer crystal, which leads to high resolution and suppresses background radiation and a Canberra $\mathrm{NaI}(T l)$ scintillation counter.

The measurements were performed with an $\mathrm{x}$-ray cell which is mounted on a two-circle goniometer. The cell is designed as a two-chamber arrangement: ${ }^{29}$ the inner chamber (height $h=65 \mathrm{~mm}$ and diameter $d=60 \mathrm{~mm}$ ) made of copper defines the surrounding of the substrate, and contains the saturated vapor atmosphere; the outer chamber guarantees the temperature stability by using a thermostated air stream which surrounds the inner chamber. The temperatures of the wetting film and of the surrounding vapor was measured every $30 \mathrm{~s}$. A glass tube furnace serves as a heat source of the cycle and a membrane pump maintains the air flow. Kapton windows in the sample cells allow the analysis with $\mathrm{x}$-ray radiation. The substrate (typical size $100 \mathrm{~mm}^{2}$ ) is mounted vertically against a thermoelectric device and placed 25 $\mathrm{mm}$ above a reservoir which contains the adsorbate $(5 \mathrm{ml})$ and defines isobaric conditions.

In order to confirm that the growth mechanism of the liquid film is independent from the experimental setup, further experiments with a different $x$-ray cell were performed. This cell was completely made out of brass, and the inside of the inner chamber was coated with HALAR $(E$-CTFE). HALAR prevents wetting and therefore assures that only the observed wafer is wetted by the adsorbate. Again the wafer was mounted in a vertical position against a thermoelectric device. Additionally a third chamber with a second thermostated air stream which surrounds the two inner chambers was added in order to obtain a higher-temperature stability. The air stream was regulated using two LAKESHORE 330 temperature controllers. These experiments yield the same results as those which were performed in the uncoated cell. Equal results were also achieved by using different types of air cycles or heating sources. This means that our results are clearly independent of experimental details.

The adsorbate is bromotrichloromethane $\left(\mathrm{CBrCl}_{3}\right)$, which is liquid at room temperature $\left(T_{m}=268 \mathrm{~K}\right.$, $T_{b}=377 \mathrm{~K}$ ). The vapor pressure (at $293 \mathrm{~K}$ ) is sufficiently high $\left(p_{g}=46 \mathrm{hPa}\right)$ to guarantee the transport of the adsorbate from the reservoir through the vapor phase to the wetting film. The structure of the $\mathrm{CBrCl}_{3}$ molecule is nearly tetrahedral, with a van der Waals diameter of 7.5 $\AA$ and a dipole moment of $p=0.21 \mathrm{D}$. Therefore the interaction between $\mathrm{CBrCl}_{3}$ and the substrate is mainly van der Waals like. The critical angle for $\mathrm{x}$-ray reflection of liquid $\mathrm{CBrCl}_{3}$ is $\theta_{c}=0.18^{\circ}$. Thus it is lower than the critical angles of all substrates used.

The substrates are as follows: (a) A polished silicon (100) wafer with a thin $(5.8 \AA)$ native oxide layer. Silicon has a high surface tension which provides the case of complete wetting. Furthermore, silicon wafers are very smooth (width of the $\mathrm{Si} / \mathrm{SiO}_{2}$ interface: $\sigma=9.5$ and $2.6 \AA$ for the rms roughness of the surface) and therefore they are well suited for X-ray reflectivity measurements. ${ }^{29}$ (b) A $p$-type doped (boron) germanium(111) wafer with a thick $(55 \AA)$ oxide layer and a quite rough $(\sigma=15 \AA)$ surface. (c) A $360-\AA$ gold and (d) a $390-\AA$ silver-film $(\sigma=26$ and $38 \AA$, respectively) evaporated on top of crownglass substrates, and (e) a bare crownglass wafer $(\sigma=12 \AA)$. Because of their high surface tension, ${ }^{30}$ a case of complete wetting is expected, too.

After the wetting experiments the bare wafers were characterized again. Only the Ag/glass wafer shows differences: Obviously chemical transformation of the silver surface happened at the beginning of the wetting experiment. The change of the critical angle indicates an additional layer of $\mathrm{AgCl} / \mathrm{AgBr}(\sigma=57 \AA)$ on top of the silver layer. Consequently the layer system glass$\mathrm{Ag} / \mathrm{AgCl} / \mathrm{AgBr}-\mathrm{CBrCl}_{3}$ was used for the fits of the wetted wafer.

\section{TIME-DEPENDENT MEASUREMENTS}

After injecting the adsorbate (liquid $\mathrm{CBrCl}_{3}$ ) into the reservoir of the $x$-ray cell, a stable wetting film with its equilibrium thickness $l_{\max }$ forms on top of the wafer. $l_{\text {max }}$ depends on the particular sample system and the temperature stability of the experimental setup $\left(\Delta T_{\infty} \approx 3\right.$ $\mathrm{mK})$. From the fit of the data we obtain values from $l_{\text {max }}=157 \AA\left(\mathrm{CBrCl}_{3}\right.$ on $\left.\mathrm{Si} / \mathrm{SiO}_{2}\right)$ up to $l_{\max } \approx 280 \AA$ ( $\mathrm{CBrCl}_{3}$ on $\mathrm{Ag} /$ glass).

The time-dependent measurements were started close to the vapor-liquid coexistence line in the $p-T$ diagram at a temperature of $T=300 \mathrm{~K}$. The disturbance of the thermodynamic equilibrium caused by a short temperature pulse $\Delta T$ between the substrate (with the wetting film of thickness $l_{\max }$ ) and the vapor phase was monitored with transverse scans (i.e., $q_{x}$ scans at a fixed position $q_{z}=0.055 \AA^{-1}$ ). Figure 3 illustrates that the sharp specular peak vanishes right after the start of the thermal disturbance. This means that the wetting film is strongly perturbed, and no longer has a well-defined surface.

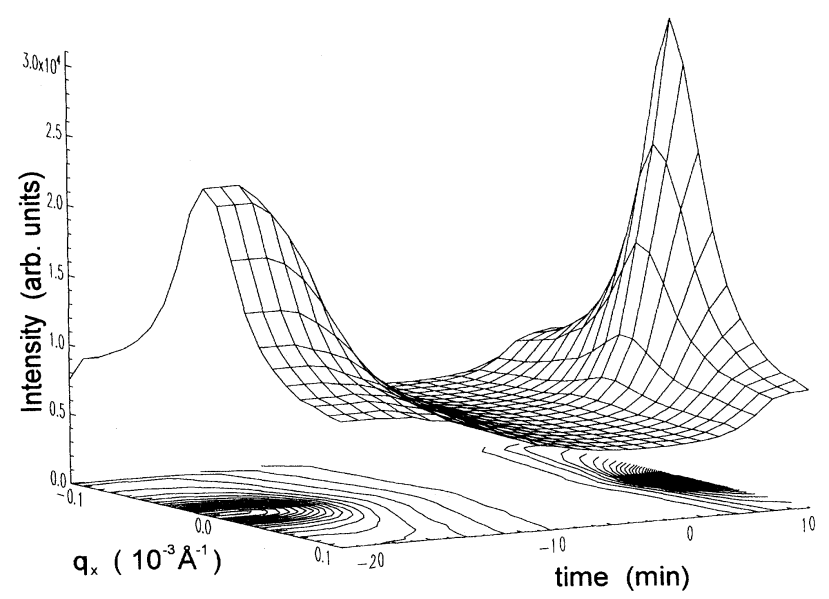

FIG. 3. Measured transverse scans $\left(q_{x}\right.$ scans at $q_{z}=0.055$ $\AA^{-1}$ ) for the system $\mathrm{CBrCl}_{3}$ on $\mathrm{Au} /$ glass over the corresponding contour plot. The evolution of the specular peak in the first half hour after the thermal disturbance can be seen. 
After switching off the heating the specular peak appears again. This means that the system begins to return to the original equilibrium state by forming a smooth but thin wetting film on top of the substrate. Subsequently the growth of this film until it reaches equilibrium thickness was monitored with time-resolved reflectivity measurements (time resolution $\Delta t=0.2-1.6 \mathrm{~h}$ ). The switch-off of the heating source defines the zero point of the time axis and the beginning of the growth. In several tests the time resolution was optimized for corresponding time constants in order to obtain both good statistics and a maximum amount of information to determine the growth parameters. As an example, Fig. 4 shows the measured reflectivities and the fit results for the system $\mathrm{CBrCl}_{3}$ on top of $\mathrm{Si} / \mathrm{SiO}_{2}$ during the first $32 \mathrm{~h}$ after the termination of the thermal disturbance. The modulation period of the reflectivities decreases with time, and consequently the thickness of the wetting layer increases. After a characteristic time (which depends on the particular system) the film remains stable and reaches the same equilibrium thickness $l_{\max }$ as before. The stability of the wetting layer was proved with additional reflectivity measurements over several hours. Due to the required time resolution the diffuse scattering was not monitored during the growth of the wetting film. However, a determination of the wetting film thickness from the fit of the $q_{z}$ scans can be obtained with high accuracy. This does not hold for the roughness $\sigma$, because a determination of this quantity requires a subtraction of the diffusely scattered intensity.

The measured time dependence of the wetting film thickness is shown in Figs. 5 (for $\mathrm{CBrCl}_{3}$ on silicon and glass) and 6 (for $\mathrm{CBrCl}_{3}$ on silver, gold, and germanium). The data were fitted with the growth models mentioned in Sec. II B (Lipowsky model, dashed lines) and Sec. II C (Kolmogorov model, solid lines), respectively. Both models can be transformed into a universal growth law, with a characteristic time constant $\tau$ :

$$
\bar{l}(t)=l_{0}+\left(l_{\max }-l_{0}\right)\left\{1-\exp \left[-(t / \tau)^{n}\right]\right\} .
$$

The dynamic exponent $n$ has to be set to $n=1$ in the case of the Lipowsky model. The results from the fits of the data are shown in Table I, which contains the time constants $\tau$ for the Kolmogorov and Lipowsky models and the thicknesses of the wetting films at the beginning $\left(l_{0}\right)$ and end $\left(l_{\max }\right)$ of the growth. The $\tau$ values differ from 1 $\mathrm{h}\left(\mathrm{CBrCl}_{3}\right.$ on silver/glass) up to $15.4 \mathrm{~h}\left(\mathrm{CBrCl}_{3}\right.$ on silicon).

\section{RESULTS AND DISCUSSION}

From Figs. 5 and 6 it can be seen that the Kolmogorov model is able to explain the data for all investigated systems, whereas the Lipowsky theory clearly fails for $\mathrm{CBrCl}_{3} / \mathrm{SiO}_{2} / \mathrm{Si}$, i.e., for systems with rather large time constants. The same holds for $\mathrm{CCl}_{4}$ on $\mathrm{Si} / \mathrm{SiO}_{2}$ (see Table II and Ref. 27). For this system with a time constant of $\tau=22 \mathrm{~h}$ the Lipowsky model fails too, whereas the more flexible Kolmogorov approach is able to explain the data with an exponent of $n=3$.

The strength of the substrate-adsorbate interaction,
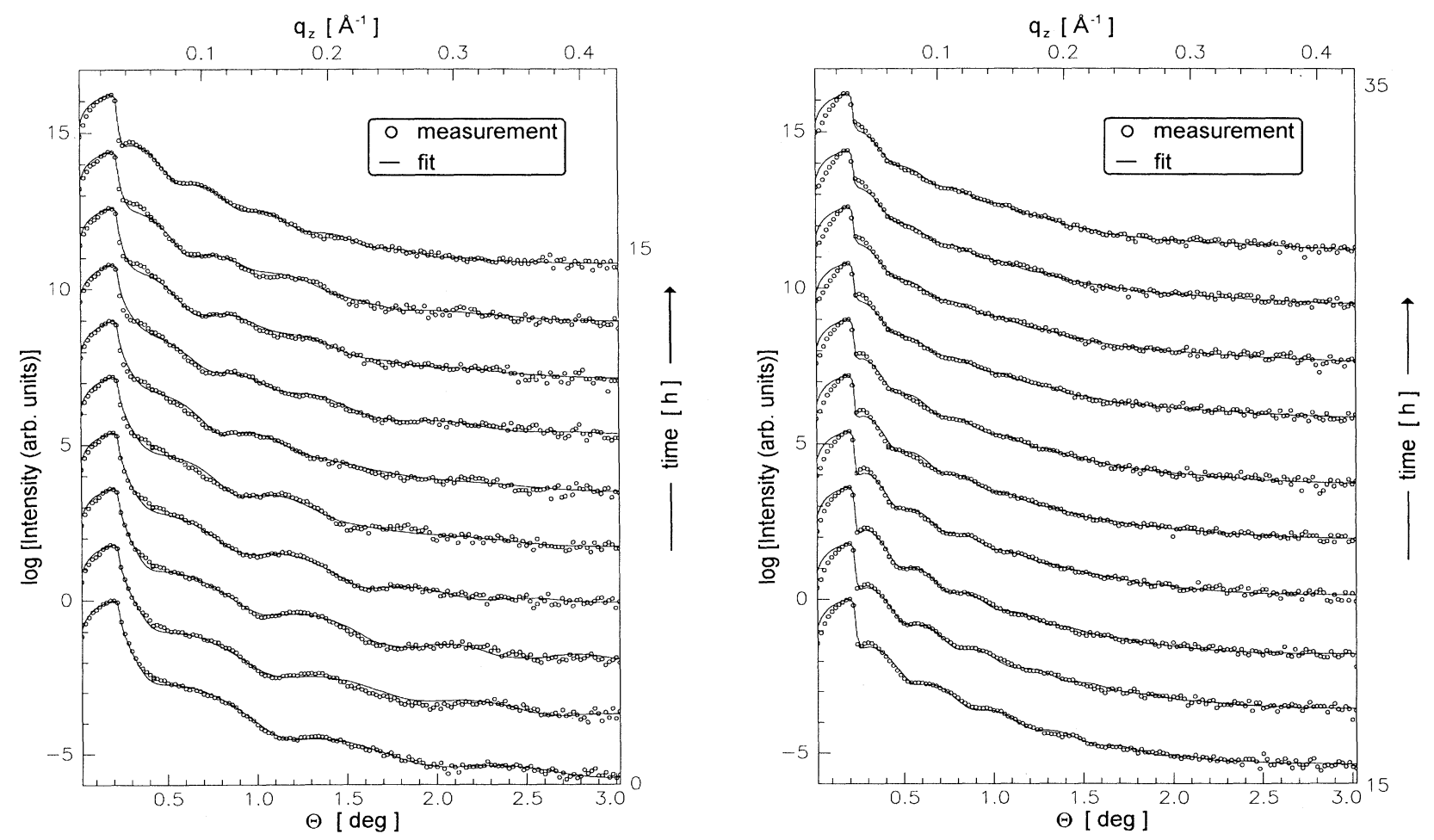

FIG. 4. Measured specular reflectivities (circles) and fit results (solid lines) for the first 16 (on the left) and the second 16 (on the right) hours. The system is $\mathrm{CBrCl}_{3}$ on top of a $\mathrm{Si} / \mathrm{SiO}_{2}$ wafer. For clarity the curves are shifted by 1.8 orders of magnitude against each other. 


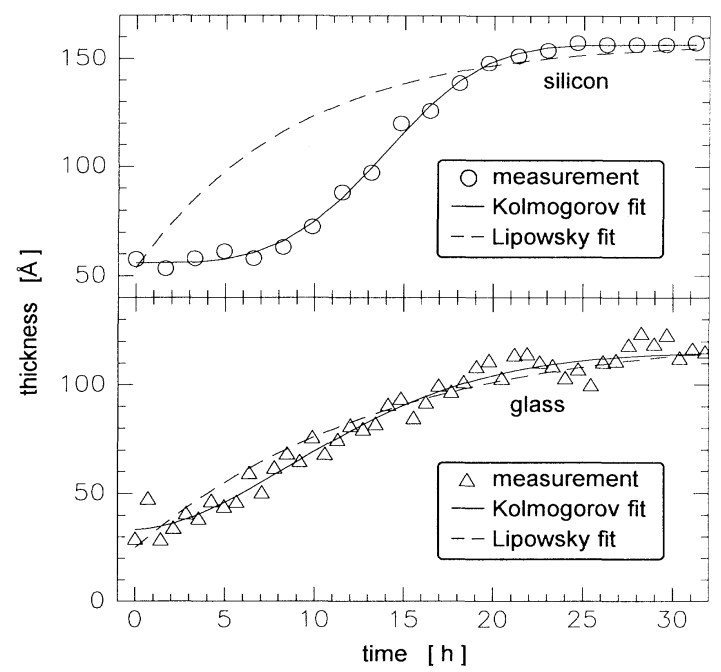

FIG. 5. Growth functions $l(t)$ of the wetting film in the systems $\mathrm{CBrCl}_{3}$ on silicon (circles) and glass (triangles). The symbol size denotes the error bars. The solid and dashed lines show the best fits using the growth models of Kolmogorov and Lipowsky, respectively.

i.e., the effective Hamaker constant, dominates the kinetic of the film evolution. A large Hamaker constant leads to a quicker film growth [see Eqs. (17) and (20)], keeping in mind that under experimental conditions the film thickness cannot become infinite. Additionally, an increase of the roughness enlarges the film thickness (at a fixed chemical potential difference $\delta \mu$ ) because the free energy is reduced. ${ }^{38}$ Therefore the roughness of the substrate can cause an acceleration of the growth process and a small value of the time constant $\tau$.

The influence of the Hamaker constant can be seen from a comparison of the growth of a $\mathrm{CBrCl}_{3}$ film on sil-

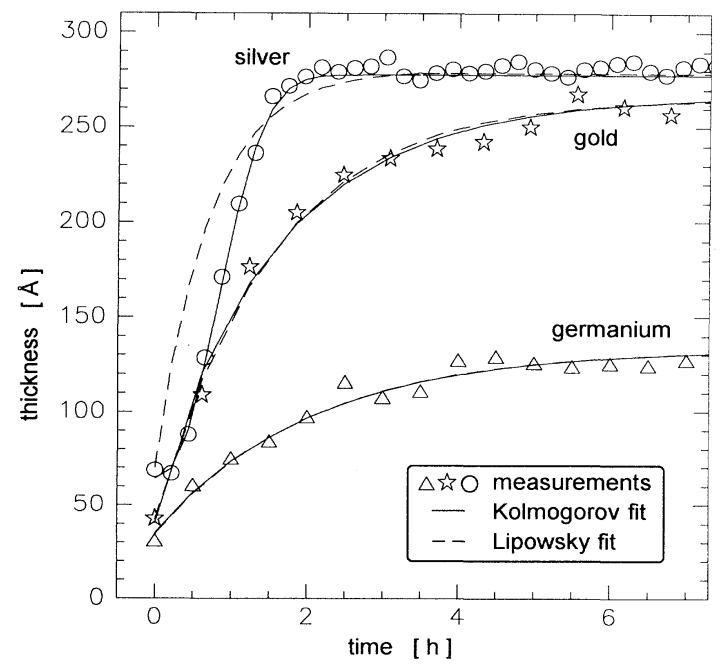

FIG. 6. Growth functions $l(t)$ of the wetting film for the systems $\mathrm{CBrCl}_{3}$ on silver (circles), gold (stars), and germanium (triangles). The symbol size denotes the error bars. The solid and the dashed lines show the best fits using the growth models of Kolmogorov and Lipowsky, respectively.
TABLE I. Results of the fits for the different substrates wetted with $\mathrm{CBrCl}_{3}$ using the Lipowsky and Kolmogorov models (Kol.) with a dynamic exponent $n$ which is free to vary in the fit, respectively. The characteristic time constant of the growth process is denoted with $\tau$. Errors (for $n$ and $\tau$ ) of approximately $5 \%$ have to be assumed. Values given in brackets indicate that the respective fit does not explain the data sufficiently.

\begin{tabular}{|c|c|c|c|c|c|}
\hline Substrate & Silicon & Germanium & Gold & Silver & Glass \\
\hline Surface roughness $\sigma(\AA)$ & 2.6 & 15 & 26 & 38 & 12 \\
\hline Lipowsky model $\tau(\mathrm{h})$ & (9) & 2.0 & 1.52 & $2 \quad(0.7)$ & 14.0 \\
\hline Kolmogorov model $\tau(\mathrm{h})$ & 15.4 & 2.02 & 1.53 & $\begin{array}{ll}3 & 1.03\end{array}$ & 313.6 \\
\hline$n($ Kol. $)$ & 3.0 & 1.0 & 0.9 & 2.3 & 1.8 \\
\hline$l_{0}(\mathrm{Kol}).(\AA)$ & 56 & 35 & 40 & 64 & 33 \\
\hline$l_{\max }($ Kol. $)(\AA)$ & 157 & 133 & 268 & 278 & 115 \\
\hline
\end{tabular}

icon with a previous experiment with $\mathrm{CCl}_{4}$ on $\mathrm{Si}^{27}$ Due to the nearly identical small roughnesses of the two $\mathrm{Si}$ substrates which were used, the difference of $7 \mathrm{~h}$ between the time constants (see Tables I and II) of $\mathrm{CBrCl}_{3}$ and $\mathrm{CCl}_{4}$ must originate from the adsorbates. Although it is difficult to measure Hamaker constants accurately, all measurements (see Refs. 29 and 31) lead to a larger Hamaker constant for $\mathrm{CBrCl}_{3}$ than for $\mathrm{CCl}_{4}$. This may explain the difference in the time constants.

The surface roughness can be described by the value of the rms roughness as well as by in-plane parameters like a correlation length $\xi$ and a Hurst parameter $h .{ }^{37}$ Due to the roughness the effective surface area is larger than the area of a smooth surface, and thus the surface is more attractive. An analysis of the off-specular x-ray scattering of the bare substrates yields their in-plane structures (see Ref. 31). Fits of the data using a fractal height-height correlation function ${ }^{37}$ yield small values of $h=0.2-0.3$ for the Hurst parameters $h$, and in-plane correlation lengths of $\xi=300-700 \AA$ for the $\mathrm{Au}$ and Ge substrates. This means that extremely jagged surfaces are present, and a purely two-dimensional growth of the wetting film is not possible. The small- $n$ values which are obtained from the data with the $\mathrm{Au}$ and Ge substrates may indicate this because in the Kolmogorov model the exponent $n$ is related to the dimension of the growth process.

Additionally the time constants $\tau$ are lowered with increased roughness. The $\tau$ value for the system $\mathrm{CBrCl}_{3}$ on glass is rather large compared with that for the $\mathrm{Ag}, \mathrm{Au}$, and Ge substrates. This cannot be reconciled with surface roughness supported film growth, but the time constant is in agreement with previous values published in the literature (see Table II). Therefore the substrate-

TABLE II. Comparison of some time constants $\tau$ for the growth dynamic of several wetting film/substrate systems.

\begin{tabular}{lc}
\hline \multicolumn{1}{c}{ System/Reference } & Time constant $\tau$ \\
\hline $\mathrm{CCl}_{4}$ on $\mathrm{Si} / \mathrm{SiO}_{2}($ Ref. 27) & $22 \mathrm{~h}$ \\
$\left(\mathrm{C}_{4} \mathrm{H}_{8}\right)_{x}-\left[\mathrm{C}_{2} \mathrm{H}_{3}\left(\mathrm{C}_{2} \mathrm{H}_{5}\right)\right]_{1-x}$ on $\mathrm{Si} / \mathrm{SiO}_{2}$ (Ref. 32) & $\approx 2 \mathrm{~d}$ \\
$\mathrm{CH}_{3} \mathrm{OH} / \mathrm{C}_{6} \mathrm{H}_{12}$ on glass (Ref. 33) & $\approx 3 \mathrm{~h}$ \\
$\mathrm{CH}_{3} \mathrm{NO}_{2} / \mathrm{CS}_{2}$ on glass (Ref. 34) & $1-10 \mathrm{~h}$ \\
$\mathrm{H}_{2} \mathrm{O}$ on quartz (Ref. 35) & $\approx 3 \mathrm{~d}$ \\
$\mathrm{SF}_{6}$ on laser mirror (Ref. 36) & $\approx 1-3 \mathrm{~d}$ \\
\hline \hline
\end{tabular}


adsorbate interaction, described by the Hamaker constant, seems to be rather small for crownglass wafers. Unfortunately no values for Hamaker constants of crownglass are published, so that this is only a speculation [even values of quartzglass cover a range of (4.2-40) $\times 10^{-20} \mathrm{~J}$ (Ref. 39)].

In general one can say that the growth rate should be small in confined systems like thin films. ${ }^{40,41}$ For ideal smooth substrates the only important value dominating the growth kinetic is the strength of the substrateadsorbate interaction. Our measurements seem to indicate, however, that the surface roughness is a second major aspect and, more specifically, the real in-plane structure (jagged or smooth). Up to now, the microscopic influence of the surface roughness of the wetting behavior of liquid films has not been well understood. Andelman, Joanny, and Robbins ${ }^{42}$ investigated how the Fourier components of a rough surface are damped by an adsorbed liquid film. But their calculations are only valid under equilibrium conditions and therefore cannot be applied to the film growth.

\section{SUMMARY AND OUTLOOK}

The growth kinetics of thin liquid wetting films thinned by a short thermal pulse disturbance was studied as a function of time for different sample systems. The growth process was monitored by $x$-ray reflectivity measurements in the region of total external reflection. The time dependence of the wetting layer thickness can be explained for several systems with the Lipowsky and for all systems with the Kolmogorov growth model.

Time constants $\tau$ in the region of hours were observed. They cover the region from $1 \mathrm{~h}\left(\mathrm{CBrCl}_{3}\right.$ on silver/glass) up to $15.4 \mathrm{~h}\left(\mathrm{CBrCl}_{3}\right.$ on silicon). Besides the different surface tensions and the adsorbate-substrate interactions, the roughnesses of the examined substrates seem to have a strong influence on the time constants $\tau$. Further measurements with other substrates are in process to investigate this topic.

In order to monitor the diffuse scattering during the growth of the wetting film, additional measurements with synchrotron radiation may be very useful. Then a separation of the nucleation rate $\Gamma$ and the growth velocity $v$ (see Sec. II C) is possible. ${ }^{27}$

\section{ACKNOWLEDGMENTS}

We want to thank K. Binder for very helpful discussions, and $\mathbf{J}$. Boneberg for the preparation of the gold and silver samples.
${ }^{1}$ P. G. de Gennes, Rev. Mod. Phys. 57, 827 (1985).

${ }^{2}$ S. Dietrich, in Phase Transition and Critical Phenomena, edited by C. Domb and J. L. Lebowitz (Academic, New York, 1988), Vol. 12.

${ }^{3}$ A. M. Cazabat, in Les Houches, Session XLVIII, 1988-Liquides aux Interfaces, edited by J. Charvolin, J. F. Joanny, and J. Zinn-Justin (Elsevier, Amsterdam, 1990).

${ }^{4}$ L. Lèger and J. F. Joanny, Rep. Prog. Phys. 55, 431 (1992).

${ }^{5}$ J. A. Nieminen, D. A. Abraham, M. Karttunen, and K. Kaski, Phys. Rev. Lett. 69, 124 (1992).

6J. F. Padday, J. Adh. Sci. Tech. 6, 1347 (1992).

${ }^{7}$ P. C. Wayner and J. Schonberg, J. Colloid Interface Sci. 152, 507 (1992).

${ }^{8}$ G. J. Hirasaki, J. Adh. Sci. Tech. 7, 285 (1993).

${ }^{9}$ R. Lipowsky, J. Phys. A 18, L585 (1985).

${ }^{10} \mathrm{~K}$. Binder, in Kinetics of Ordering and Growth at Surfaces, Vol. 239 of NATO Advanced Study Institute, Series B: Physics, edited by M. G. Lagally (Plenum, New York, 1990).

${ }^{11}$ I. M. Tidswell, T. A. Rabedeau, P. S. Pershan, and S. D. Kosowsky, Phys. Rev. Lett. 66, 2108 (1991).

${ }^{12}$ L. B. Lurio, T. A. Rabedeau, P. S. Pershan, I. A. Silvera, M. Deutsch, S. D. Kosowsky, and B. M. Ocko, Phys. Rev. Lett. 68, 2628 (1992).

${ }^{13}$ L. G. Parrat, Phys. Rev. 95, 359 (1954).

14J. Lekner, Theory of Reflection (Nijhoff, Dodrecht, 1987).

${ }^{15} \mathrm{R}$. W. James, The Optical Principles of the Diffraction of $X$ rays (Oxbow, Woodbridge, 1962).

${ }^{16}$ W. A. Hamilton and R. Pynn, Physica B 173, 71 (1991).

${ }^{17}$ S. M. Heald, H. Chen, and J. M. Tranquada, Phys. Rev. B 38, 1016 (1988).

${ }^{18}$ A. Schalchli, J. J. Benattar, and C. Licoppe, Europhys. Lett. 26, 271 (1994).
${ }^{19}$ R. Lipowsky, Phys. Rev. Lett. 52, 1429 (1984).

${ }^{20}$ J. H. Awbery, A University Text-book of Physics, Vol. III, Heat (Griffin, London, 1952); I. Newton, Philos. Trans. R. Soc. London 22, 824 (1701).

${ }^{21}$ A. N. Kolmogorov, Izv. Akad. Nauk SSSR Ser. Mater. 3, 355 (1937).

${ }^{22}$ E. S. Johnson and R. F. Mehl, Trans. Am. Inst. Min. Metal. Pet. Eng. 135, 416 (1939).

${ }^{23}$ M. J. Avrami, J. Chem. Phys. 7, 1103 (1939).

${ }^{24}$ M. J. Avrami, J. Chem. Phys. 8, 212 (1940).

${ }^{25}$ M. J. Avrami, J. Chem. Phys. 9, 177 (1941).

${ }^{26}$ N. Hamaya, Y. Yamada, J. D. Axe, J. D. Belander, and S. M. Shapiro, Phys. Rev. B 33, 7770 (1986).

${ }^{27}$ P. Müller-Buschbaum, M. Strzelczyk, M. Tolan, and W. Press, Z. Phys. B 98, 89 (1995).

${ }^{28}$ L. Brügemann, R. Bloch, W. Press, and M. Tolan, Acta Crystallogr. Sec. A 48, 88 (1992).

${ }^{29}$ P. Müller-Buschbaum, M. Tolan, and W. Press, Z. Phys. B 95, 331 (1994).

${ }^{30}$ CRC Handbook of Chemistry and Physics, 66th ed. (Chemical Rubber, Boca Raton, FL, 1986).

${ }^{31}$ M. Strzelczyk, Master's thesis, Universität Kiel, 1995.

${ }^{32}$ U. Steiner, J. Klein, E. Eisen, A. Budkowski, and L. J. Fetters, Science 258, 1126 (1992).

${ }^{33}$ P. Guenoun and D. Beyens, Phys. Rev. Lett. 65, 2406 (1990).

${ }^{34}$ X. I. Wu, D. Ripple, and C. Franck, Phys. Rev. A 36, 3975 (1987).

${ }^{35}$ R. M. Pashley and J. A. Kitchener, J. Colloid Interface Sci. 71, 491 (1979).

${ }^{36}$ M. R. Moldover and R. W. Gammon, J. Chem. Phys. 80, 528 (1984); R. F. Kayser, M. R. Moldover, and J. W. Schmidt, J. Chem. Soc. Faraday Trans. 2 82, 1701 (1986). 
${ }^{37}$ S. K. Sinha, E. B. Sirota, S. Garoff, and H. B. Stanley, Phys. Rev. B 38, 2297 (1988).

${ }^{38}$ M. O. Robbins, D. Andelman, and J. F. Joanny, Phys. Rev. A 43, 4344 (1991).

${ }^{39}$ J. Visser, Adv. Colloid Interface Sci. 3, 331 (1972).
${ }^{40}$ H. Orihara and Y. Ishibashi, J. Phys. Soc. Jpn. 61, 1919 (1992).

${ }^{41}$ E. Bosco, J. Chem. Phys. 97, 1542 (1992).

${ }^{42}$ D. Andelman, J. F. Joanny, and M. O. Robbins, Europhys. Lett. 7, 731 (1988). 


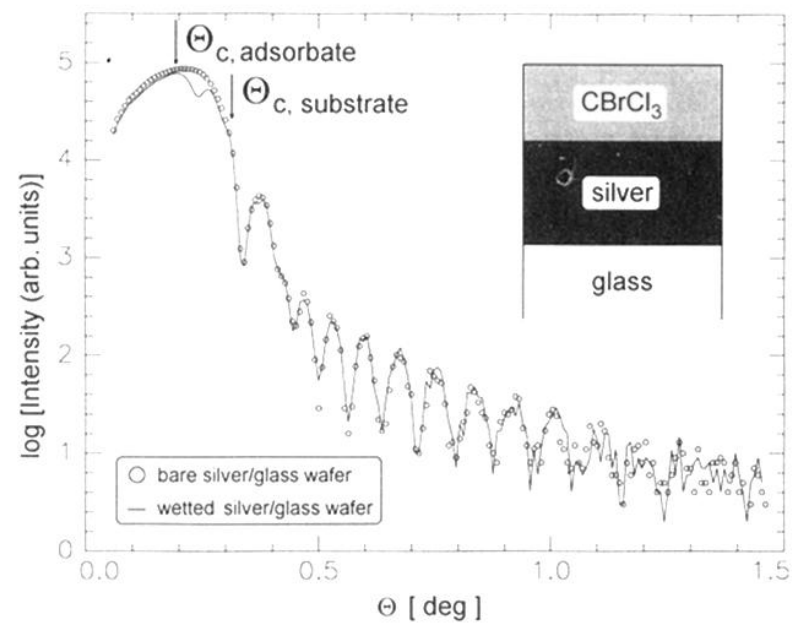

FIG. 1. Measured specular reflectivities of a bare (open circles) and a wetted (solid line) silver/glass wafer. For incident angles $\Theta_{\text {dip }}$, which fulfill the condition

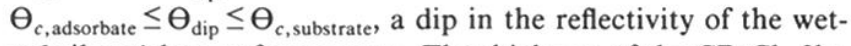
ted silver/glass wafer appears. The thickness of the $\mathrm{CBrCl}_{3}$ film is about $280 \AA$. The inset schematically shows the layer system. 


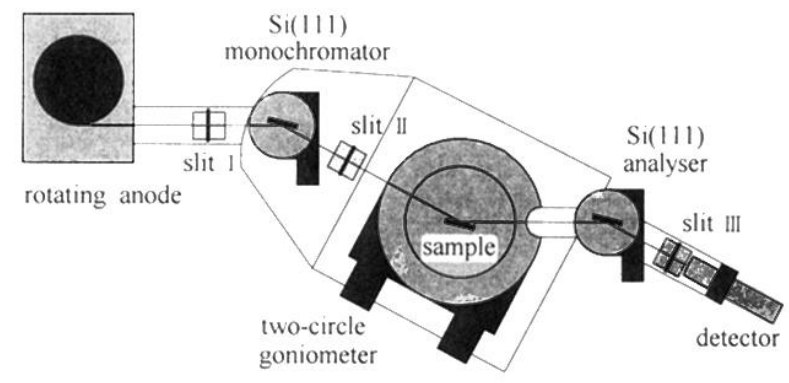

FIG. 2. Setup of a high-resolution three-crystal diffractometer (TCD) with a silicon analyzer and monochromator crystal. 